\title{
Propiedades esenciales o necesidad hipotética de las causas: Kripke y Aristóteles
}

\author{
Ángel Martínez Sánchez ${ }^{1}$
}

Recibido: 16 de octubre de 2017 / Aceptado: 17 de octubre de 2018

\begin{abstract}
Resumen: Saul Kripke ha presentado un conjunto de tesis esencialistas enmarcadas bajo la etiqueta «esencialismo aristotélico», esto es, la tesis que defiende la significatividad de la distinción entre propiedades necesarias y accidentales de los objetos. Sin embargo, ¿es el «esencialismo aristotélico» acorde con el aristotelismo? Más aún, ¿puede alguna de las propiedades esenciales presentadas por Kripke ser interpretadas desde una perspectiva aristotélica? En este artículo pretendemos fundamentalmente dos cosas: 1) criticar la etiqueta «esencialismo aristotélico» a través de una hipótesis plausible acerca de su elaboración; y 2) aportar una explicación de las intuiciones que sustentan la metafísica kripkeana desde la metafísica aristotélica, centrada en el paralelismo existente entre la necesidad del origen material de los artefactos y la doctrina de la necesidad hipotética de la causa material desarrollada por el filósofo de Estagira.
\end{abstract}

Palabras clave: Kripke, Aristóteles, necesidad del origen material de los artefactos, esencialismo aristotélico, necesidad hipotética, causa.

\section{[en] Essential properties or hypothetical necessity of causes: Kripke and Aristotle}

Abstract: Saul Kripke has defended a set of essentialist thesis under the so called «Aristotelian essentialism» (the claim that it makes sense to characterize objects as having necessary and accidental properties). However, is «Aristotelian essentialism» genuinely Aristotelian? Is it possible to interpret Kripke's essential properties in Aristotelian terms? In this paper we pretend to: 1) criticize the label «Aristotelian essentialism» trying to explore its origins; 2) explain which are the intuitions that support Kripke's metaphysical framework from an Aristotelian view, focusing on the parallelism between the necessity of material origin of artifacts and the hypothetical necessity of material cause.

Key words: Kripke, Aristotle, the necessity of material origin of artefacts, «Aristotelian essentialism», hypothetical necessity, cause.

Sumario: 1. Introducción; 2. La necesidad del origen material de los artefactos en el proyecto esencialista kripkeano; 3. Propiedades esenciales, causalidad y necesidad hipotética; 3.1. Predicación per se y propiedades esenciales; 3.2. La necesidad del origen material frente al aristotelismo; 3.2 .1 Doctrina general de las cuatro causas; 3.2.2 La necesidad hipotética de la causa material: Physica, B, 9; 4. Conclusiones; 5 . Referencias bibliográficas.

$1 \quad$ Universidad Católica San Antonio de Murcia amartinez@ucam.edu ORCID: 0000-0002-6915-808X 
Cómo citar: Martínez Sánchez, A. (2019): "Propiedades esenciales o necesidad hipotética de las causas: Kripke y Aristóteles", en Revista Anales del Seminario de Historia de la Filosofía 36 (1), 221-241.

\section{Introducción}

El esencialismo contemporáneo se construye bajo el supuesto de que es significativo dividir las propiedades de re de un individuo en propiedades necesarias y propiedades accidentales: esto es, entre propiedades que un sujeto no puede dejar de tener sin dejar de ser él mismo y propiedades que un sujeto puede tener o no tener sin dejar de ser él mismo. Esta idea, presentada bajo la etiqueta de «esencialismo aristotélico», ha pasado a constituir un lugar común en el espectro de la filosofía analítica de nuestros días. En efecto, cuando uno piensa hoy en el «esencialismo aristotélico» es esta distinción la que se tiene en mente y se señala a Aristóteles como el primero en haberla establecido. De igual modo, y por extensión, se relaciona la noción de esencia con las propiedades necesarias de un individuo. Por ejemplo, Chistopher Kirwan afirmaba lo siguiente en uno de los Proceedings of the Aristotelian Society: "La palabra-raíz «esencia» puede hoy ser definida como «la suma de las propiedades esenciales». Así, «la Geidad es (completamente) la esencia de $x$ » significa « $x$ es esencialmente $G$ y para todo $H$, si $x$ es esencialmente $H$, es una verdad necesaria que las $G$ s son $H \gg$ "2. En otras palabras, que hoy hay que entender que la esencia de Calias es el conjunto de sus propiedades necesarias (sive esenciales): la esencia de Calias es algo que Calias tiene.

Los desarrollos teóricos expuestos en Naming and Necessity y en Identity and Necessity por el filósofo norteamericano Saul Kripke engarzan con esta manera de entender la esencia ${ }^{3}$. En efecto, después de haber defendido la inteligibilidad de la distinción entre propiedades esenciales y accidentales, mediante la crítica a la noción de mundos posibles en su relación con la cuestión de identidad transmundana, y, tras la diferenciación entre nociones de índole epistemológica y nociones de índole metafísica, Kripke inicia su incursión en una serie de tesis esencialistas no triviales. Estas tesis suponen la explicitación de dos formas de necesidad de re para individuos, esto es, en el esquema kripkeano, de la ejemplificación de dos tipos de propiedades que son necesariamente verdaderas de los objetos en todo mundo posible en el que dichos objetos existan: por un lado, la necesidad del origen material de los artefactos $\mathrm{y}$, por otro, la necesidad del origen biológico de los vivientes. No olvidemos que, al

2 "The root-word «essence» may now be defined as «the sum of essential properties». Thus «Gness is the (whole) essence of $x »$ means « $x$ is essentially $G$ and for any $H$, if $x$ is essentially $H$, it is a necessary truth that $G$ s are H»"; Kirwan, Сн. "How strong are the objections to essence?". Proceedings of the Aristotelian Society, vol. 71, 1970-1971, p. 44.

3 Kripke, S. Naming and Necessity, Harvard, Cambridge, 1980; originalmente publicado como Kripke, S. "Naming and Necessity" en Davidson D., Harman G. (eds.). Semantics of Natural Languages, Reidel, Dordrecht, 1872, pp. 253-355. Citaremos siempre por la reedición de la versión de 1980: Kripke, S. Naming and necessity, Blackwell, Oxford, 2013 y usamos la traducción de Kripke, S., El nombrar y la necesidad, tr. Margarita M. Valdes, Universidad Autónoma de Mexico, Mexico, 2005. A partir de ahora, $N \& N$. Kripke, S. "Identity and Necessity", en Munitz, K., Milton K. (eds.). Identity and Individuation, New York University Press, New York, 1971, pp. 135-164. Citaremos por su reedición en Kripke, S. Collected papers, vol. 1, Philosophical troubles, Oxford University Press, Oxford, 2013, pp. 1-26; usamos como traducción Kripke, S. "Identidad y necesidad", tr. Margarita M. Valdes, Cuadernos de Crítica, nº 7, Universidad Autónoma de México, nº 7, México, 1978,47 pp. A partir de ahora, $I \& N$ 
enmarcar esta disquisición en el marco del problema genuino de la transidentificación, lo que se persigue es un criterio ontológico que permita justificar la mismidad de un objeto a través de los mundos: ¿cómo es posible que un sujeto siga siendo el mismo a pesar del cambio?, esa es la pregunta pertinente. Kripke encuentra en la noción clásica de esencia, entendida, desde la perspectiva del «esencialismo aristotélico», como propiedad esencial, una vía para responder a esta cuestión.

Sin embargo, pensar que la esencia es una propiedad o un conjunto de propiedades de la substancia es, desde una perspectiva aristotélica, un error. Esto es algo que, como veremos, se desprende de las tesis defendidas en los libros centrales de la Metafisica. Dado que esta cuestión precisaría de un amplio desarrollo, en este artículo pretendemos poner en diálogo a Kripke con Aristóteles, centrándonos en la formulación de la necesidad del origen material de los artefactos, enmarcándola en el problema general del «esencialismo aristotélico» propugnado por el propio Kripke. Por ello, y desde un punto de vista formal, tratamos a continuación los siguientes puntos: 1) presentamos, de manera sintética, la denominada necesidad del origen material de los artefactos en el marco general del esencialismo analítico contemporáneo; 2) intentamos justificar el posible origen de la etiqueta «esencialismo aristotélico» y lanzamos una hipótesis acerca de por qué se ha pensado que esta manera de entender la esencia es de raigambre aristotélica; 3) defenderemos que esta manera de entender la esencia es radicalmente ajena al filósofo de Estagira; 4) finalmente, plantearemos que la necesidad del origen material de los artefactos puede ser considerada análoga, no a la cuestión aristotélica de la esencia, sino a doctrina de la necesidad hipotética de la causa material desde un análisis de Physica B, 9.

\section{La necesidad del origen material de los artefactos en el proyecto esencialista kripkeano}

Para introducir por vez primera la noción de propiedad esencial, Kripke recurre a la consideración de la constitución material originaria de los artefactos. Es decir, dado un artefacto cualquiera, parece que la procedencia material del mismo le es necesaria. Esto es, dicho artefacto, una vez construido, no podría haber sido hecho a partir de una materia distinta. De tal modo que, parece inteligible decir que, el conjunto material que está en el origen de un artefacto es una propiedad esencial del mismo. Recordemos el célebre ejemplo del atril de madera:

He aquí un atril. Una pregunta que con frecuencia ha surgido en filosofía es: ¿Cuáles son sus propiedades esenciales? ¿Qué propiedades, aparte de algunas triviales como identidad consigo mismo, son tales que este objeto tiene que tenerlas si existe de alguna manera, y si el objeto no las tuviera, no sería este objeto? Por ejemplo, podría ser una propiedad esencial de este atril el estar hecho de madera, y no de hielo. Tomemos solamente el enunciado más débil, que no está hecho de hielo; éste lo establecerá de manera tan vehemente, tal vez dramática, como sea necesario. Suponiendo que este atril esté realmente hecho de madera, ¿podría haber sido hecho este mismísimo atril desde el principio de su existencia, de hielo, digamos, de agua congelada del Támesis? Uno tiene la impresión de que no podría; aunque de hecho uno podría haber elaborado ciertamente un atril con aguas del Támesis, congelarlo mediante algún procedimiento y colocarlo exactamente aquí en lugar de esta cosa. Si alguien hubiera hecho eso, habría hecho, por 
supuesto, un objeto diferente; no habría sido este mismísimo atril y, así, no tendríamos un caso en el que este mismo atril, aquí presente, había sido hecho de hielo o había sido hecho de agua del Támesis ${ }^{4}$.

Vemos, por tanto, que Kripke trata de mostrar mediante este ejemplo lo que entiende por propiedad esencial. Dado este atril, fabricado de esta madera, parece imposible para este atril el no haber sido construido de esta mismísima madera.

Sin embargo, el planteamiento de la cuestión puede ser confuso. Así dicho, parecería que Kripke viene a concluir que es una propiedad esencial del atril el no ser de agua congelada del Támesis. Por ello, él mismo reconoce que esta es la versión más débil del planteamiento ${ }^{5}$. No obstante, es claro que la intención de Kripke no es la de aferrarse a esta versión débil como una posición canónica, sino como un instrumento para constatar nuestras intuiciones acerca de la necesidad de re. Comprender, en efecto, que si este atril hubiese sido fabricado a partir de otro conjunto material no sería este mismísimo atril, sino otro diferente, parece suscitar la idea positiva de que la mismísima materia de la cual fue originado le es esencial una vez ese objeto individual ha sido ya constituido. Además, así se insiste en la idea de que las propiedades esenciales que tienen que ver con el origen material de los artefactos sólo son accesibles por la vía del conocimiento empírico y, por tanto, se muestra que el esencialismo precisa como condición la total distinción entre lo necesario, lo contingente, lo a priori y lo a posteriori ${ }^{6}$. En efecto, erigiendo la materia particular de la que está hecha un objeto $x$ como propiedad necesaria y esencial de ese $x$ individualizado, Kripke está proclamando el acceso a un ámbito de propiedades necesarias de los objetos únicamente cognoscible a posteriori. Dado este atril, reconozco que está hecho de esta madera, y un atril, otro atril, podría haber sido hecho con otra materia, con otra madera, de acero, de carbono puro... Ningún análisis independiente de la experiencia podría hacernos descubrir la particular materia de la que está hecho o no este mismísimo atril. Tendríamos, así, un caso de necesidad de re, no a priori.

Por tanto, la intuición de Kripke es que la materia a partir de la cuál un objeto ha sido hecho es esencial a ese objeto. Si tuviéramos dos mesas construidas a partir de diversos conjuntos materiales, por muy semejantes que estas fuesen, incluso aunque pudiéramos fabricarlas de tal modo que fuesen indiscernibles desde el punto de vista observacional, serían dos mesas distintas ${ }^{7}$. Por tanto, digámoslo de forma positiva, si P es la materia específica que está en el origen de un objeto, el condicional $\langle P \rightarrow \square P »$

\footnotetext{
I\&N, pp. 15-16. También, vid. N\&N, III, pp. 113-115.

De todos modos, esto no evita que podamos denunciar lo desafortunado del ejemplo. No poseer una propiedad determinada no es, de ninguna manera, una propiedad esencial de nada. Una propiedad esencial es una propiedad que un objeto no puede no tener sin dejar de ser dicho objeto y, por tanto, es una propiedad que positivamente se tiene. Si aceptamos la posibilidad de no poseer una propiedad como algo esencial a los objetos estaríamos diciendo que las propiedades esenciales de los objetos son infinitas y, además, no diríamos nada acerca de qué se cumple del objeto, sino lo que no se cumple. Si, dado este atril, es necesario de él el no haber sido construido a partir de cualquier otro conjunto material, y consideramos que eso es una propiedad esencial, también debería ser necesario de él el no haber sido construido de hielo, ni de cobre, ni de carbono puro, ni de hierro, ni de ese otro trozo de madera, etc. Esta es una cuestión muy antigua. Sin ir más lejos, todo esto recuerda a la crítica general desarrollada por Aristóteles contra aquellos que niegan el principio de contradicción: vid. Met. $\Gamma, 4$, 1007 a $1-10$.

$I \& N$, p. 16.

$N \& N$, III, pp. 114-115.
} 
es necesariamente verdadero. Además, dado que el conocimiento del antecedente sólo es empíricamente accesible, la conclusión $(\square P)$, alcanzable mediante modus ponens, se trata de una verdad necesaria cognoscible a posteriori $i^{8}$. En resumen, si un objeto tuvo su origen en un determinado conjunto material, no podría haber tenido su origen en ningún otro trozo de materia. Por ello, Kripke termina por introducir la tesis positiva: la materia de la que está hecho algo le es esencial, es una propiedad necesaria que ese objeto tiene .

Vemos, por tanto, cómo Kripke encuentra en la noción de esencia una vía para señalar las condiciones necesarias de la mismidad de los individuos. En efecto, la pregunta clave es: ¿qué propiedades no puede no tener un individuo sin dejar de ser sí mismo? De este modo, si $x$ es un objeto en el mundo actual e $y$ es otro objeto en un mundo posible, $x$ e $y$ serán el mismo objeto, si y sólo si $x$ e $y$ comparten las mismas propiedades esenciales. En este caso concreto, si $x$ es una mesa del mundo actual e $y$ una mesa en otra situación contrafáctica, $x$ e $y$ serán la misma mesa si y sólo si la materia de la que $x$ e $y$ fueron hechas es una y la misma. Así, las propiedades esenciales tienen como misión principal la de asegurar la mismidad ontológica de los objetos.

Sin embargo, de aquí debemos resaltar una serie de cuestiones subyacentes a esta ontología. La esencia es entendida como las propiedades que un objeto no puede no tener sin dejar de ser él mismo, pero dichas propiedades no son identificadas con el sujeto. En otras palabras, la esencia no es lo que el sujeto es, sino algo que el sujeto tiene. Por lo tanto, la esencia no está pensada al modo aristotélico, según el cual la esencia es algo que constituye al objeto en cuanto tal, sino como aquellas propiedades que le son necesarias para su mismidad. Pero entonces, ¿qué es el objeto? Parece que la ontología kripkeana ofrece un panorama en el que el objeto es un puro «esto», el mismísimo esto que fue hecho de esta o aquella materia.

Es precisamente esta deriva, contraria al aristotelismo la que nos ha hecho pensar que la etiqueta «esencialismo aristotélico», defendida por el propio Kripke, es ajena al pensamiento del Estagirita. En lo sucesivo, criticaremos, en primer lugar, la etiqueta «esencialismo aristotélico» desde el aristotelismo, para sugerir ulteriormente un puente entre dicho esencialismo y las doctrinas del propio Aristóteles.

\section{Propiedades esenciales, causalidad y necesidad hipotética}

\subsection{Predicación per se y propiedades esenciales}

Consideramos que la creencia de que la distinción entre propiedades esenciales y propiedades accidentales, a la que se alude bajo la etiqueta «esencialismo aristotélico», se debe a una descuidada interpretación ontológica de la distinción

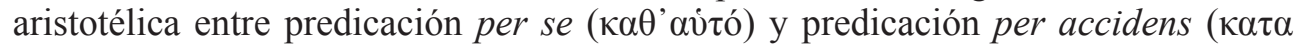
$\sigma v \mu \beta \varepsilon \beta \eta \kappa o ́ \varsigma)$. Vamos a intentar reconstruir una hipótesis acerca de este asunto ${ }^{10}$.

$I \& N$, p. 16-17.

$N \& N$, III, n. 57, p. 114.

10 Desde la aparición de la voz «esencialismo aristotélico» han aparecido numerosos trabajos que han pretendido explicar tanto sus orígenes como su distancia con respecto al pensamiento genuino de Aristóteles. Algunos de estos trabajos son: White, N. "The origins of Aristotle's essentialism", Review of Metaphysics, $\mathrm{n}^{\circ}$ 26, 1972, 
En el capítulo cuarto del libro Z de la metafísica, Aristóteles abre el examen lógico ( Allí podemos leer:

Y, en primer lugar, hagamos acerca de él algunas aclaraciones de carácter lógico, a saber, que la esencia de cada cosa es lo que se dice que ésta es per se ( $\kappa \theta^{\prime}$ 'avंó). Pues tu esencia no es el ser músico; pues no eres músico en cuanto eres tú mismo. Lo es por consiguiente, lo que eres en cuanto tú mismo ${ }^{11}$.

La introducción del examen lógico de la esencia supone el primer estadio en la investigación de la misma. Su principal objetivo, antes del enfoque real ( $\varphi v \sigma ı \kappa \tilde{\omega} \varsigma$ $\sigma \kappa о \pi \varepsilon i v)$, es la de mostrar el nexo estructural existente entre un tipo concreto de

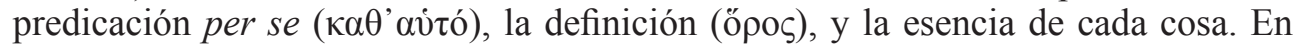
efecto, la esencia ha de poder ser predicable per se de cada cosa. Sin embargo, una lectura aislada de este fragmento puede inducir a error. Pues, si bien es cierto que toda definición expresa lo que es predicable per se de un sujeto, no todo lo que es predicable per se es definitorio del sujeto ni expresa su esencia. En otras palabras, la noción de per se es mucho más amplia que la de esencia.

En el libro $\Delta$ de la Metafísica, Aristóteles distingue tres sentidos fundamentales de per $s e^{12}$. En primer lugar, se dice per se la esencia de una cosa. Así, "Calias es por sí mismo Calias y la esencia de Calias"13. En segundo lugar, se dice per se todo lo que lo que entra en el qué es ( $\tau$ $\varepsilon \dot{\delta} \sigma \tau v)$. Por ello, predicamos «animal» de Calias, pues «animal» es una de las partes de su definición ${ }^{14}$. Pero, en tercer lugar, también son predicables per se todas aquellas propiedades o características que incluyen necesariamente al sujeto en sus enunciados y que, por ende, no pueden pertenecer a ningún otro. Ahora bien, pese a que estas propiedades pertenecen necesariamente a un sujeto o a alguna de sus partes, no entran en su definición. Así, «par» e «impar» se predican per se del número, o «blanco» de una superficie, dadas las características propias de la materia a partir de la cual fue fabricada, o macho y hembra de animal ${ }^{15}$.

Esta triple distinción es la que hace que, al nivel de la estricta predicación, Aristóteles divida las proposiciones en cuatro familias de predicables. Las tres primeras son formas de predicación per se que vienen a corresponderse con los sentidos que acabamos de enumerar, el cuarto es la predicación per accidens: definición (őpos), género $(\gamma \varepsilon \dot{v} v \varsigma)$ y propio ('íıv), por un lado, y accidente ( $\sigma u \mu \beta \varepsilon \beta \eta \kappa o ́ \varsigma)$, por otro. En primer lugar, la definición es el enunciado que significa la esencia de una substancia:

pp. 57-85; Cohen, S. Marc. "Essentialism in Aristotle”, Review of Metaphysics, n 31, 1978, pp. 387-405; Kung, J. "Aristotle on Essence and Explanation", Philosophical Studies, no 31, 1977, pp. 361-383; Witt, Ch. "Aristotelian Essentialism Revisited”, Journal of the History of Philosphy, n 27-2, 1989, pp. 285-298.

11 Met. Z, 4, 1029 b 13-16. Para la traducción al español de la metafísica utilizo la edición trilingüe de Valentín García Yebra, (Aristóteles. Metafísica, ed. trilingüe de Agustín García Yebra, Gredos, Madrid, 1998.) pero en algunos casos modifico la traducción para ajustarme mejor al texto griego.

12 Lo que encontramos en el capítulo 18 del libro $\Delta$ de la Metafísica es la enumeración de cinco sentidos. No obstante, según Giovanni Reale, al que seguimos en este punto, el cuarto y el quinto son reducibles al primero y al tercero. Vid. Reale, G. Introduzione, traduzione e comentario della «Metafísica» di Aristotele, Bompiani, Milano, 2009, p. 951; ibid., p. 1011. Cfr. Met. $\Delta, 18,1022$ a 24-36

13 Met. $\Delta, 18,1022$ a $26-27$.

14 Vid. Met. $\Delta, 18,1022$ a 27-29; cfr. An. Post. A, 73 a 35.

15 Vid. Met. $\Delta, 18,1022$ a 29-32; cfr. An. Post. A, 73 a 38-73 b 3; Met. Z, 4, 1029 b 16-18. 
v.g., «animal racional» supone la definición de hombre ${ }^{16}$. En segundo lugar, el género es lo que se predica dentro del qué es, acerca de varias cosas que difieren en especie: v.g., «animal» con respecto al tigre y al caballo ${ }^{17}$. En tercer lugar, el propio es lo que no indica la esencia pero se da sólo en tal objeto y puede intercambiarse con él en la predicación: v.g., es propio del hombre el ser capaz de aprender gramática y, por ello, si es capaz de aprender gramática será un hombre y si es un hombre será capaz de aprender gramática ${ }^{18}$. Por su parte, accidente es lo que no es ni definición, ni género, ni propio pero se da en un objeto. En otras palabras, el predicable accidente indica lo que puede darse y no darse en un mismo sujeto, como "ser músico» con respecto al hombre ${ }^{19}$.

Nótese que aquí aparece una posible dificultad interpretativa entre la definición y el propio. En el propio, aquello que se predica del sujeto le pertenece de forma tal que puede sustituirlo en el orden de la predicación. En efecto, lo mismo da decir hombre que «ente capaz de aprender gramática». Sin embargo, alguien podría observar que lo mismo sucede con la definición. Pues, análogamente, lo mismo es «animal racional» que hombre. Entonces, ¿qué diferencia hay? Para responder esta cuestión de forma positiva tendríamos que introducirnos de lleno en la teoría de la definición. Pero esto bien merecería un tratamiento independiente. Conformémonos con responder de forma indirecta con un argumentum ad verecundiam. En efecto, lo cierto es que, para Aristóteles, hay claramente una diferencia que obliga a distinguirlos pese a tener esta conducta común en la predicación. Esta diferencia radica en que la definición expresa la esencia, el propio, no:

Ahora bien, toda proposición y todo problema indican, bien un género, bien un propio,
bien un accidente (pues también la diferencia, al ser genérica, ha de ser colocada en el
mismo lugar que el género); y, ya que entre lo propio lo hay que significa la esencia y lo
hay que no, se ha de dividir lo propio en las dos partes antedichas, y a una se la llamará
definición, que significa la esencia, y al a otra, de acuerdo con la designación dada en
común a ambas, se la llamará propio, Así, pues, es evidente, a partir de lo dicho, por
qué, de acuerdo con la presente división, todo viene a reducirse a cuatro cosas: propio,
definición, género o accidente ${ }^{20}$.

Por tanto, la construcción de la idea de que se debe a Aristóteles el denominado «esencialismo aristotélico», puede ver su origen en una precipitada identificación entre definición y predicación per se. Esto es, que la totalidad de las cosas que son predicables per se de un sujeto son definitorias de él. Además, es muy plausible que dicha identificación haya sido propiciada por la traducción de algunos términos aristotélicos en el espectro cultural anglosajón. Por ejemplo, es usual encontrar predicación per se y predicación per accidens traducidos respectivamente por essential predication y accidental predication ${ }^{21}$. Si a este modo de traducir, le

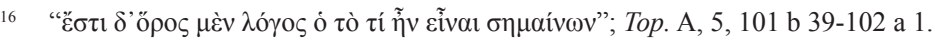

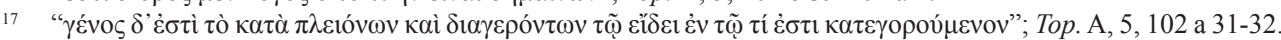

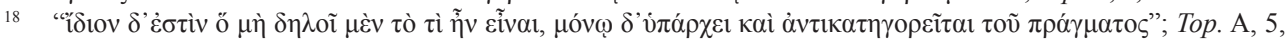
102 a $18-19$.

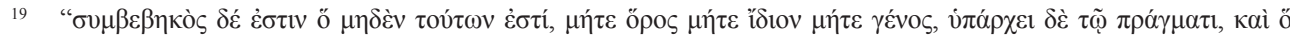

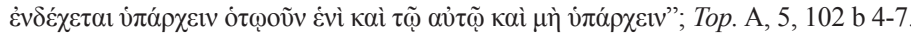

20 Top. A, 4, 101 b 18-26.

21 Charlotte Witt hace la misma apreciación en: Witt, CH. Substance and essence in Aristotle: an interpretation of “Metaphysics VII-IX”, Cornell University Press, New York, 1989, p. 105. De igual modo, la autora advierte que
} 
añadimos una cierta tendencia a no diferenciar los planos predicativo y ontológico en la obra aristotélica, alguien puede convertir con facilidad lo que supone una distinción entre dos modos de predicar en dos grandes familias de propiedades de los objetos: propiedades esenciales (essential properties) y propiedades accidentales (accidental properties). A quién haya operado así, no le resultará difícil pensar en el «esencialismo aristotélico» como aquella doctrina que distingue entre las propiedades esenciales y las propiedades accidentales de los objetos y como aquella para la cual, las propiedades esenciales, todas ellas, son las que constituyen la esencia de cada cosa. En efecto, dado que Aristóteles pretende en Z 4 expresar la relación que existe entre un individuo y su esencia desde una perspectiva lógica, podría parecer plausible pensar en la esencia como el conjunto de todas las propiedades que son predicables per se de dicho individuo. Además, Aristóteles indica que todo lo que es predicado de los sujetos en cuanto tales les pertenece necesariamente: "las cosas que se dicen en sí en cuanto que se dan dentro de los predicados o en cuanto que los predicados se dan en ellas son por sí mismas y por necesidad"22. En definitiva, ¿por qué no identificar la esencia con las propiedades necesarias de los individuos?

Como ya hemos mostrado, la mera existencia de la diferenciación entre la definición y el propio indica que, para Aristóteles, la respuesta a la pregunta qué es algo no consiste en la predicación sistemática del conjunto de propiedades necesarias de un sujeto. Además, justo después del pasaje que hemos citado de Z 4 , Aristóteles se apresura a limitar la noción de predicable per se a aquellos casos en los que lo predicado es lo que el sujeto es, su definición y no a sus propiedades necesarias:

Pero tampoco todo esto (todo lo que pertenece al sujeto en cuanto tal), pues no lo es lo que es en cuanto tal como lo blanco para una superficie, porque la esencia de una superficie no es la esencia de lo blanco. Pero tampoco el compuesto de ambos, la esencia de una superficie blanca, porque se añade lo mismo que se define ${ }^{23}$.

Esto es, aquello a lo que viene a hacer referencia la definición, es a lo que es la cosa misma, desprovista no sólo de las propiedades que le pertenecen accidentalmente sino también de aquellas que le pertenecen per se y con razón de necesidad. Cualesquiera de estas propiedades predicadas de los sujetos no expresan lo que los sujetos son sino que expresan las propiedades que se inhieren a ellos, por adición.

Esto es lo que impide que las propiedades necesarias de los objetos, o afecciones $(\pi \alpha ́ \theta \eta)$, no puedan ser definitorias de las substancias, pues las nociones correspondientes a estas propiedades precisan incluir aquello que se define. Pongámoslo en términos generales. Imaginemos una substancia $a$ y una propiedad necesaria suya $F$. ¿Podemos decir qué es a partir de su propiedad $F$ ? La respuesta aristotélica es negativa, porque esto convierte el enunciado en circular. ¿Por qué? Porque la propiedad necesaria de un objeto precisa siempre ser predicada de su

también ha podido influir en todo este malentendido la traducción del predicado proprium por la voz inglesa property (propiedad): vid. ibid., p. 106, n. 6. Un ejemplo claro de esta tendencia en las traducciones inglesas lo encontramos en las muy extendidas ediciones de la Loeb Classical Library y la Clarendon Aristotle Series: Aristotle. Posterior Analytics, Topica, traducidos respectivamente por Tredennick, H. y Forster, E.S., ed. Loeb, Harvard University Press, Massachusetts 1960; Aristotle. Topics. Books I and VIII with excerpts from related texts, translated with a commentary by Robin Smith, Clarendon Press, Oxford, 1997; y Aristotle. Posterior Analytics, translated with a commentary by Jonathan Barnes, Clarendon Press, Oxford, 1994, entre otros.

22 An. Post. A, 4, 73 b 16-19.

23 Met. Z, 4, 1029 b 16-19. 
sujeto. De tal modo que si al preguntar qué es $a$ respondemos $F a$ lo definido entra en la definición y se nos abre la posibilidad del típico argumento ad infinitum: pues $F a$, sería $F F a$, y a su vez, $F F F a$, etc. Podemos instanciar esta forma argumentativa con uno de los ejemplos propuestos por el propio Aristóteles: si femenino es una afección necesaria de animal y pretendemos que femenino sea definitorio de animal, dado que la noción de femenino será siempre la de «animal femenino» nos veremos forzados a asumir que animal es «animal femenino», y, a su vez, que animal es «animal animal femenino», ad infinitum ${ }^{24}$. Dicho de otro modo, dado que las propiedades no pueden existir separadas de sus sujetos necesariamente se han de remitir a ellos, tanto en el plano de la predicación como en el real. En efecto, no existe lo femenino sino como metáfora, sino un animal que es femenino. Por tanto, ninguna propiedad, por muy necesaria que sea, está capacitada para ser la esencia ni puede ser definitoria sensu stricto.

Así pues, "el enunciado en que no esté lo que se define, y que sin embargo lo enuncie, ése será el enunciado de la esencia de cada $\operatorname{cosa}{ }^{25} \mathrm{y}$, por tanto, el enunciado no podrá enunciar una propiedad no separable de la cosa, sino que expresará lo que es el sujeto en cuanto tal. Por tanto, dado que las propiedades son segundas con respecto a su sujeto y siempre se remiten a él, la definición ha de ser la expresión de algo primero, no de algo que se inhiere al sujeto, sino del sujeto mismo. Dicho bajo la forma de un lema: la definición debe expresar al sujeto de propiedades, no las propiedades del sujeto. Así, no es que haya un sujeto cuya su esencia sea el conjunto de las propiedades necesarias que él tiene, sino que el sujeto y su esencia son uno y esto será algo determinado per se, separado, algo primero y principio de las propiedades:

Pues la esencia es lo mismo que el qué es; y, cuando una cosa se dice de otra, no es algo determinado ( $\tau o ́ \delta \varepsilon \tau \imath$ ); por ejemplo, el hombre blanco no se identifica con algo individual, si la individualidad corresponde sólo a las substancias; de suerte que sólo habrá esencia de aquellas cosas cuyo enunciado es una definición. Y no es definición si un nombre significa lo mismo que un enunciado (...), sino únicamente si es de algo primero ( $\pi \rho \tilde{\omega} \tau \tau$ tov $\tau$ ); y son tales las cosas que se dicen no porque una se diga de otra ${ }^{26}$.

Y por ello, dado que algo será primero únicamente si no implica la referencia a ninguna otra cosa (como en hombre-blanco, blanco refiere a hombre, y en animalfemenino, femenino refiere a animal), Aristóteles concluye: "No habrá, pues, esencia

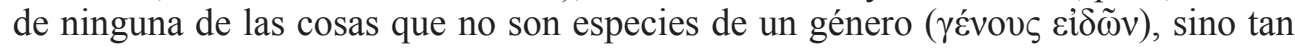
sólo de éstas (pues éstas parecen decirse no por participación ni como afección ni como accidente)" ${ }^{27}$. En efecto, esto primero sólo podrá ser la especie última de un género, la especie que emana de una diferencia última.

24 Esta misma forma argumentativa es explotada de forma recursiva en el célebre ejemplo de la nariz, la chatez y la concavidad, en Z 5. Vid. Met., Z, 4, 1030 b 14-28; ibid. 1030 b 28-1031 a 1. Hemos evitado este ejemplo en concreto, pues es cierto que puede ser considerado un ejemplo desafortunado. Es decir, alguien podría poner en duda que la chatez pueda constituir una propiedad necesaria de una nariz. Sin embargo, como bien han indicado Frede y Patzig, masculino o femenino son características que pertenecen sólo al animal y por ello constituye un ejemplo más apropiado: Frede, M., Patzig, G. Il libro Z della Metafisica di Aristotele, Vita e pensiero, Milano, 2001, p. 236.

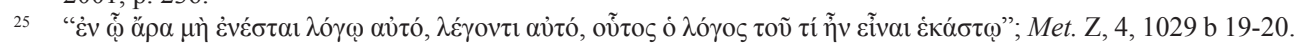

26 Met. Z, 4, 1030 a 3-11.

27 Met. Z, 4, 1030 a 11-14. 
En suma, hasta aquí hemos visto dos cosas. En primer lugar, hemos propuesto una posible hipótesis acerca de cómo es posible que se haya tildado de aristotélico al esencialismo contemporáneo. Nuestra reconstrucción indica que es plausible que se deba, por un lado, a una interpretación en términos de propiedades de la distinción aristotélica entre predicación per se y predicación per accidens. Por otro lado, esto ha favorecido la creencia de que la esencia está constituida por el conjunto de las propiedades necesarias de los objetos. En segundo lugar, hemos propuesto algunos argumentos por los que puede verse que esta posición es ajena al pensamiento aristotélico ${ }^{28}$.

Ahora bien, llegados a este punto alguien podría decir: "nada de esto afecta a Kripke, pues las propiedades necesarias a las que él se refiere no son de la misma naturaleza que aquellas a las que se refiere Aristóteles". Esto es totalmente cierto. Sin embargo, esto no impide que lo dicho sea aplicable a Kripke de forma crítica. Pues, si lo que pretendemos hacer es una lectura aristotélica del esencialismo kripkeano, lo que de aquí se desprende es que Kripke llama propiedades a cosas que para Aristóteles no son propiedades en sentido estricto. Ahora bien, ¿qué son? ¿Existe algún puente interpretativo entre las propiedades kripkeanas y el esquema aristotélico? Lo hay. Si preguntásemos a un aristotélico recalcitrante qué es para esta mesa la mismísima madera con la que fue hecha, creo que sin necesidad de muchos quebraderos de cabeza respondería: "es evidente, es la causa material de la mesa". En efecto, aquí, lo que para Kripke es una propiedad esencial de los objetos para Aristóteles es un tipo de causa ${ }^{29}$.

Por otro lado, el modo en que Kripke plantea la cuestión de las propiedades esenciales, como propiedades necesarias, nos obliga a examinar este asunto no desde la perspectiva de las causas en general sino desde el punto de vista de las causas necesarias. Lo curioso es que en este examen descubriremos que, en primer lugar, las propiedades necesarias ven su análogo en un tipo de necesidad muy concreta dentro del esquema aristotélico y, en segundo lugar, que el hecho de que esté basada en ese tipo de necesidad hace que el conocimiento de dicha necesidad sea necesariamente $a$ posteriori. En otras palabras, si tenemos éxito, aportaremos una justificación de las intuiciones que soportan el esencialismo kripkeano desde el aristotelismo.

28 Decimos «algunas» porque podrían alegarse otras muchas. Por ejemplo, si aceptáramos que las propiedades necesarias de los objetos fueran la esencia de cada cosa, dado que todas las cosas poseen propiedades necesarias, todas las cosas serían substancias. Sin embargo, una vez más, todo esto es extraño al pensamiento de Aristóteles. Para él, un número o un brazo no son substancias, ni tienen esencia, ni son definibles sensu stricto. Sin embargo, eso no impide que puedan poseer propiedades que les pertenezcan en cuanto tales. Una vez más, la identificación entre propiedades necesarias y esencia es de difícil encaje.

29 Charllotte Witt discurre casi del mismo modo en el último capítulo de su Substance and essence in Aristotle, en el que compara esquemáticamente el esencialismo kripkeano con el pensamiento de Aristóteles, pero abarcando todos los tipos de propiedades esenciales (la necesidad el origen material de los artefactos, la necesidad del origen biológico de los vivientes y la necesidad de la constitución interna de las especies) defendidos por Kripke en relación con las cuatro causas: Witt, Ch. op. cit., pp. 188-189. Consideramos que la observación de Witt no sólo es correcta, sino especialmente evidente tras la lectura de la célebre nota 57 de Naming and Necessity (N\&N, III, n. 57, pp. 114-115). Tanto es así, que muchos de los grandes especialistas en Kripke han propuesto hablar de «relación necesaria» en lugar de «propiedad necesaria» (Pérez Otero, M. Esbozo de la filosofía de Kripke, Montesinos, Barcelona, 2015, p. 162; Forbes, G. The Methaphysics of Modalitiy, Clarendom Press, Oxford, 1985, pp. 150-153). Sin embargo, analizar por completo los paralelismos existentes entre Kripke y Aristóteles excede en mucho los límites de un único artículo. 


\subsection{La necesidad del origen material frente al aristotelismo}

\subsubsection{Doctrina general de las causas}

Para Aristóteles, el mundo de la experiencia, el mundo fenoménico, se nos presenta como algo dado, bajo el esquema de un conjunto de existencias efectivas, de realidades sujetas al cambio. Dada la tendencia humana al conocimiento, en sentido general ( $\tau$ ò $\varepsilon i \delta \delta ́ v a l)$, el hombre se enfrenta cognitivamente con los fenómenos bajo las diversas formas de conocimiento de las que es capaz ${ }^{30}$.

Ahora bien, con respecto al mundo fenoménico, el hombre no se contenta con un saber que radique en la mera constatación de aquello que precisamente le es dado, sino que aspira a un discurso que sea realmente justificativo. En efecto, los fenómenos nos son dados pero conocerlos es buscar aquello que los justifica, aquello en virtud de lo cual algo ha devenido una existencia concreta. En definitiva, conocer

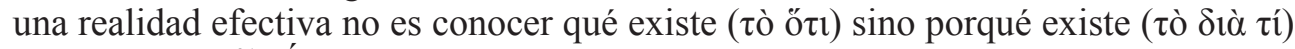
eso que existe ${ }^{31}$. Éste es el marco general de la doctrina aristotélica acerca de las causas. El verdadero saber consiste en la delimitación del porqué y ese porqué no es otra cosa que la causa primera ( la apertura de Physica, B, $3^{32}$.

Como es harto conocido, la indagación etimológica indica que, en sus orígenes, el término griego de causa ( $\alpha i$ íía) tenía dos significados fundamentales: acusación o imputación, por un lado y culpa, por otro. Tras el uso traslaticio de estos conceptos por la filosofía, el término incluye los sentidos técnicos de causa y razón ${ }^{33}$. Por

30 En el primer capítulo del libro alpha de la Metafísica Aristóteles lleva a cabo lo que podríamos llamar una fenomenología del saber, esto es, un examen de las diferentes manifestaciones o formas del saber humano.

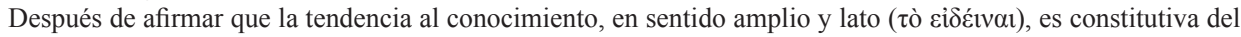
género humano (Met., A, 1, 980 a 21), Aristóteles distingue seis formas generales de saber agrupables en tres familias: la percepción ( $\alpha$ ǐ $\sigma \eta\rceil \varsigma \varsigma)$, la imaginación ( $\varphi \alpha ́ v \tau \alpha \sigma \mu \alpha)$ y el recuerdo ( $\mu \nu \eta ́ \mu \eta)$, por un lado; la experiencia ( $\dot{\varepsilon} \mu \pi \varepsilon 1 \rho i ́ \alpha)$, por otro, y, finalmente, el arte $(\tau \dot{\varepsilon} \chi v \eta)$ y la ciencia $(\dot{\varepsilon} \pi \imath \tau \eta \dot{\mu} \mu \eta)$. Acto seguido, explica la naturaleza de cada una de ellas desvelando que cada forma supone un grado más en escala del conocimiento con respecto a la que le precede. Sobre este asunto y en relación con la Metafísica como ciencia de los primeros principios y causas, vid. García-Lorente, J. A. "La ciencia de los principios y de las causas primeras", Anales del Seminario de Historia de la Filosofia, vol. 33, n 1, 2016, pp. 11-31."

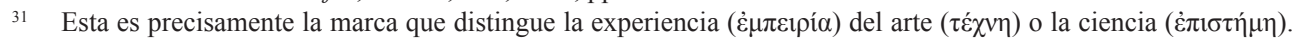

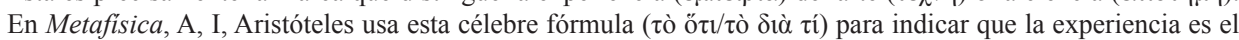
mero conocimiento de lo que acaece de forma particular, pero que el arte, que este aspecto es semejante a la ciencia, conoce universalmente la causa que está presente una potencial multiplicidad de casos: "Creemos, sin embargo, que el saber y el entender pertenecen más al arte que a la experiencia, y consideramos más sabios a los conocedores del arte que a los expertos, pensando que la sabiduría corresponde en todos al saber. Y esto, porque

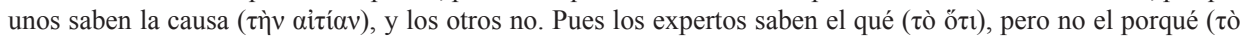

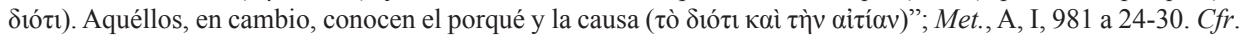
ibid. A, 1, 981 a 7-12; ibid. A, 1, 981 a 12-16.

32 Phys. B, 3, 194 a 16-23, donde causa primera se refiere aquí a causa próxima, es decir, aquella realidad efectiva que es inmediatamente responsable de un efecto.

33 Natali, C. "Aitia in Plato and Aristotle, from everyday language to technical vocabulary", en Natali, C., Viano, C., Zingano, M. (ed.), Aitia I, Les quatre causes d'Aristote. Origines et interpretation, Editions Peeters SA, Leuven, Belgium, 2013, p. 39. En realidad el vocabulario filosófico griego este tipo de nociones tomadas del habla ordinaria. Algunos de estos ejemplos son especialmente resaltables. Por ejemplo, el profesor García Marqués ha explorado este anclaje del lenguaje filosófico en el habla ordinaria en lo tocante a los sintagmas

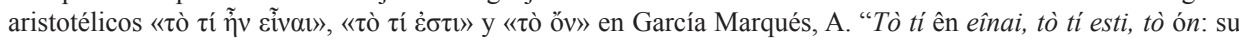
sentido y traducción", Convivium, 2017 (en prensa). 
tanto, buscar las causas de un ente es buscar aquellas realidades, todas ellas, que son «culpables» o «responsables» de su existencia efectiva ${ }^{34}$. La idea aristotélica es que, para dar una explicación suficiente de un existente en acto, el que investiga deberá preocuparse de buscar todas y cada una de las realidades responsables de un mismo objeto, si no la explicación resultará insatisfactoria.

No hay duda alguna de que uno de los grandes logros de Aristóteles ha sido el de agrupar todas las realidades, en los términos de su eficacia causal, en cuatro

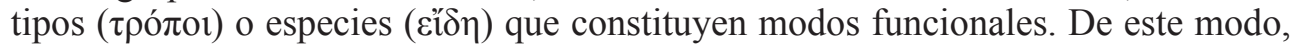
podemos clasificar las causas no en orden al tipo de realidades que son (pues éstas son potencialmente infinitas) sino según su función, según el papel que desempeñan en la justificación de una existencia. Así, Aristóteles elabora la célebre doctrina de las cuatro causas, de las cuatro funciones causales del cambio:

En un sentido se llama causa a aquello a partir de lo cual ( $\tau$ ò $\dot{\varepsilon} \xi$ oṽ) se origina algo

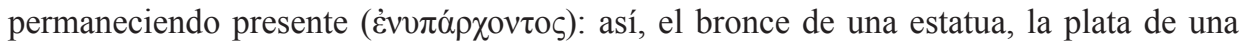
copa y sus géneros respectivos; en otro sentido, se llama causa a la forma y al modelo

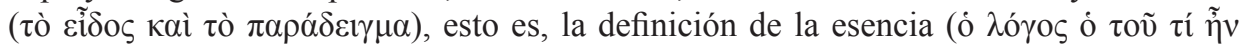

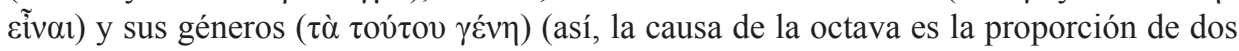
a uno, y en general el número) así como las partes que entran en la definición. Aún más,

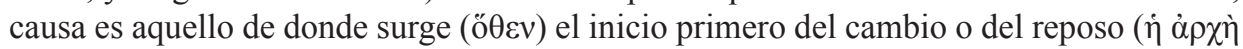

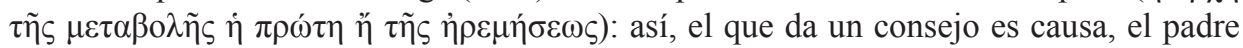
es causa del hijo y, en general, lo que hace es causa de lo que es hecho y lo que cambia

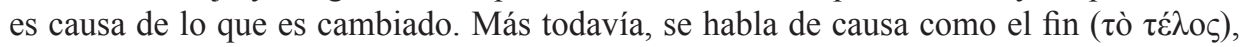

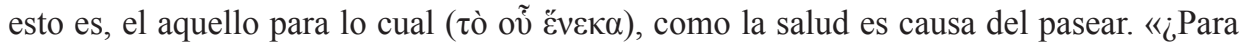
qué pasea?». Decimos: «para estar saludable» y diciendo así creemos haber aducido la causa $^{35}$.

Como puede observarse, Aristóteles no se limita a acuñar un término para cada familia causal, sino que utiliza de modo recurrente un conjunto de sintagmas nominales. Este recurso tiene la finalidad de mostrar que las cuatro causas no son cuatro entes, sino cuatro papeles que una realidad puede desempeñar en orden a la explicación de un fenómeno. Tenemos como primera especie de causa, el aquello a partir de lo cual ( $\tau$ ò $\dot{\varepsilon} \xi$ oũ) se origina algo, a la que en Metafísica A se refiere con

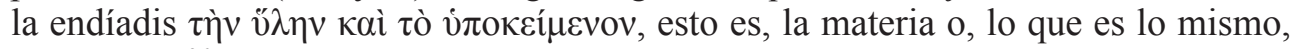
el substrato ${ }^{36}$. Se trata de la causa material, la materia desde la que algo es hecho y

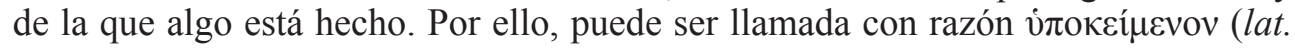
substratum: lo extendido por debajo), pues su función es la de acoger la forma que viene a darle una estructura determinada. Así, el bronce es causa material de una estatua, pues desde el bronce se hace $\mathrm{y}$, una vez hecha, es de bronce ${ }^{37}$.

34 Y decimos, todas ellas, porque forma parte de la argumentación aristotélica la idea de que la tradición filosófica precedente no había conseguido elaborar el esquema completo de la tipología de las causas, si bien las había dilucidado todas pero de forma segregada, aislada o insuficiente. En efecto, este es el sentido en el que, en los capítulos centrales de Metafísica A (cap. 3-10), Aristóteles evalúa las doctrinas de los filósofos anteriores al trazar una historia de la filosofía centrada en la noción de causa.

35 Phys. B, 3, 194 a 23-35; cfr. con el texto casi literal Met., $\Delta, 2,1013$ a 24-35 y con Met. A, 3, 983 a 24-34, texto posterior en el que Aristóteles se remite a la Física como la obra en la que la doctrina de las causas ha sido ya suficientemente expuesta.

36 Met., A, 3, 983 a 29-30.

37 Cfr. Berti, E., Rossitto, C. Aristotele. Il libro primo, Laterza\&Figli Spa, Roma-Bari, 1993, p. 72. Sobre el 


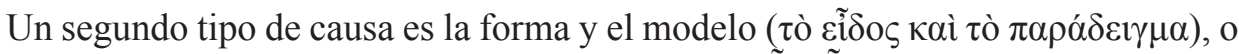

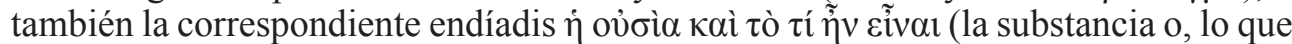
es lo mismo, el qué era ser) ${ }^{38}$. A través de estos sintagmas, Aristóteles se refiere a la causa formal, esto es, a aquello que hace que algo sea lo que es. De ahí, la referencia directa a la definición. Responder al porqué de algo también consiste en delatar que es aquello que hace que algo sea lo que de hecho es, y esto ve su expresión en la

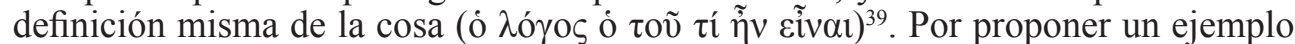
que no dé lugar a duda: el alma racional es la causa formal de un hombre y, al hombre, lo definiremos como animal racional.

En tercer lugar, el aquello de donde surge (ö $\theta \varepsilon v)$ el inicio primero del cambio o

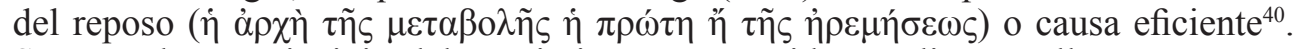
Se trata de un principio del movimiento, en sentido amplio, aquello en acto que inicia el proceso que llevará a algo que aún no es a ser algo en acto. En este caso, el ejemplo de Aristóteles es preclaro: el padre es la causa eficiente en lo que respecta a la generación del hijo ${ }^{41}$.

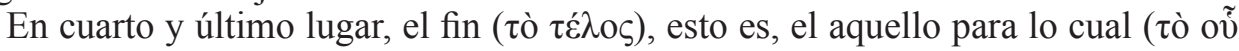

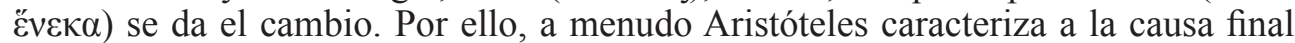
como opuesta a la eficiente. Si esta supone el principio activo del cambio, la causa formal supone el término, el momento último ( $\tau$ ò $̌ \sigma \sigma \chi \alpha \tau o v)$, aquello en vistas a lo que se da el movimiento ${ }^{42}$. También es común ver como Aristóteles considera el fin de un proceso como el bien ( en sentido moral, sino que el fin es «bueno» por coincidir con el estado perfecto y acabado, con la realización completa de una $\operatorname{cosa}^{43}$. Así, la causa final del paseo es la salud, pues quien pasea lo hace para tener salud.

Es importante señalar que Aristóteles no pretende decir que hayamos de encontrar estas cuatro funciones causales en la explicación de toda existencia efectiva. De hecho, hay existencias en el universo aristotélico que obviamente carecen de algunas de ellas: v.g. los motores carecen, por definición, de causa eficiente, material y final, sino que son pura forma ${ }^{44}$; de igual modo, los eventos que son fruto de la fortuna ( $\tau \dot{\chi} \chi \eta)$ o la espontaneidad ( $\alpha$ vó́ $\mu \alpha \tau o v$ ), adolecen de la posesión de una causa final propia $^{45}$. Lo que aquí se pretende advertir es que causa se dice en cuatro sentidos ( $\tau \dot{\alpha} \delta^{\prime} \alpha^{\prime \prime} \tau 1 \alpha \lambda \varepsilon^{\prime} \gamma \varepsilon \tau \alpha \iota \tau \varepsilon \tau \rho \alpha \chi \tilde{\omega} \varsigma$ ) y que estos sentidos suponen cuatro funciones que son irreductibles las unas a las otras. De tal modo que, dado un fenómeno que es considerado como objeto de estudio, aquel que investiga habrá de examinar qué realidades desempeñan en cada caso cada una de estas funciones, esto es, qué es aquello que hace las veces de materia, de forma, de agente eficiente y de fin.

recurso Aristotélico de la endíasis como elemento retórico: ibid. p. 71; $c f r$. García-Lorente, J. A. "La ciencia de los principios y de las causas primeras", Anales del Seminario de Historia de la Filosofía, vol. 33, n 1, 2016, pp. 24-25.

38 Met., A, 3, 983 a 27-28.

39 Cfr. Berti, E., Rossitto, C. Op. cit., pp. 71-72.

40 La única diferencia con el texto paralelo de Metafísica A radica en que Aristóteles elige la fórmula más general

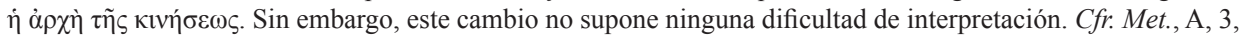
983 a 30.

$41 \quad$ Cfr. Berti, E., Rossitto, C. Op. cit., p. 72.

42 Phys. B, II, 194 a 28-33; Met., A, 3, 983 a 31.

43 Met., A, 3, 983 a 32. Cfr. Berti, E., Rossitto, C. Op. cit., p. 72. Cfr. Berti, E. Aristotele. Dalla dialettica alla filosofia prima con saggi integrative, Bompiani, Milano, 2014, pp. 385-386.

44 Met., $\Lambda, 6-7$.

45 Phys., B, 4-6. 
De igual forma, el esquema aristotélico permite considerar una cosa en general e indicar sus causas generales, así como descender al nivel de los individuos, en el cuál se fundan nuestras consideraciones generales. De esta manera podemos decir, por ejemplo, que la causa de la estatua es el escultor, pero de la Pietà, Michelangelo Bounarroti; del hombre en general, otro hombre, pero "Que Peleo lo es (causa) de Aquiles, y de ti, tu padre" 46 . Sin embargo, se debe insistir en que nuestra capacidad de considerar en general los objetos y sus causas no debe dar la impresión de que Aristóteles crea que en la existencia de causas universales. Como hemos dicho, las causas son especies funcionales que se instancian, por así decirlo, en cada caso particular. Por ello, Aristóteles advierte: in re, "las causas y principios de cosas diferentes son (...) diferentes" y que sólo "Si se habla universal y analógicamente, son las mismas para todas las cosas" $"$. Pero, como bien ha indicado Berti, decir que las causas de cosas diversas son idénticas por analogía es lo mismo que decir que, in re, son diversas en número y en especie pero que las aunamos, in mente, bajo un mismo tipo de relación ${ }^{48}$. En efecto, ningún hombre individual tiene las mismas causas que otro hombre. Ahora bien, considerados en cuanto al concepto, todos los hombres tienen las mismas causas: "Para las cosas que están dentro de la misma especie son diferentes (las causas), no específicamente, sino porque son diferentes las causas de los individuos: tu materia y tu especie y tu causa motriz y la mía, aunque en su concepto universal sean las mismas"49. En definitiva, sólo las existencias individuales tienen realmente causas y esas causas serán siempre causas únicas.

Una vez planteadas las líneas generales de la doctrina de las cuatro causas, volvamos nuestra mirada al esencialismo kripkeano. Parece claro que la denominada propiedad esencial del origen material ve su correspondencia con la causa material. Sin embargo, la gran diferencia radica en que, para Aristóteles, las causas no suponen, al menos en su conjunto, la esencia del individuo. La coordinatio causarum va dirigida a la justificación, tanto en el orden gnoseológico como en el ontológico, de la existencia del individuo. Ante la pregunta, ¿por qué existe $x$ ?, alegamos las causas y éstas nos proporcionan la explicación suficiente de una existencia individual. Pero justificar la existencia de algo no es lo mismo que indicar la esencia. Dicho con un ejemplo parco, decir que una taza ha sido hecha de loza no quiere decir que la esencia de la taza sea esa mismísima porción de loza, ni siquiera una parte de su esencia. Lo que sí tiene sentido decir es que esta mismísima porción de loza de la que está hecha esta taza es una de las causas de esta taza.

En suma, si antes decíamos que ninguna propiedad puede ser la esencia de un individuo y que esta creencia, por parte del esencialismo contemporáneo, se debía a una determinada interpretación ontológica de la predicación per se, ahora indicamos que lo que Kripke denomina propiedades esenciales son las causas responsables de la existencia de un objeto. Sin duda, el hecho de que no todo pueda tener las mismas causas, si no es por analogía, está detrás de esta confusión terminológica del esencialismo kripkeano. Cuando la investigación va dirigida al individuo concreto, sus causas son únicas, son causas de ese individuo y, si nos fuera dado otro individuo,

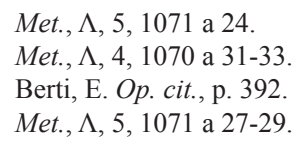


sus causas serían necesariamente diversas. Creemos que ésta es la razón por la que Kripke ha pensado en los términos de un conjunto de propiedades necesarias lo que realmente son causas. O, lo que es lo mismo, ha pensado las causas como propiedades que si fueran distintas el individuo sería necesariamente otro.

Ahora bien, Kripke califica a estas propiedades como necesarias de los objetos. Vamos a ver a continuación la relación que existe en Aristóteles entre necesidad y causalidad, para terminar de señalar una vía que explique las intuiciones que sostienen el esencialismo kripkeano desde una perspectiva puramente aristotélica.

\subsubsection{La necesidad hipotética de la causa material: Physica, B, 9.}

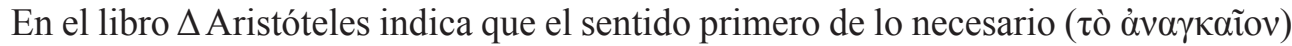
es "lo que no puede ser de otro modo" ". Tras eso, introduce una distinción entre dos tipos generales de necesidad que se distinguen en función del origen causal de la misma: "Así, pues, algunas cosas tienen en otra la causa de que sean necesarias, pero otras no, sino que a causa de éstas son necesarias otras" ${ }^{1}$. Esto es, tenemos, por un lado, la denominada necesidad simpliciter ( $\dot{\alpha} \pi \lambda \tilde{\omega} \varsigma)$ si la causa de lo necesario se debe a algo intrínseco ${ }^{52}$. Dicha causa supondrá entonces una causa necesaria y suficiente de un determinado proceso. Así, por ejemplo, si la materia es por sí misma causa de la corrupción de los entes, será verdadero que «necesariamente el hombre es mortal», por la sola necesidad de la materia de la que está hecho el compuesto. En segundo lugar, tenemos la necesidad secundum quid o $\dot{\varepsilon} \xi$ vं de la necesidad se debe a algo extrínseco. Eso necesario será, con respecto a su causa externa, una condición necesaria pero no suficiente del proceso en cuestión ${ }^{53}$. Así, por ejemplo, si se da la casa, necesariamente se dan los cimientos: a $\rightarrow \square$ b. En este caso, la necesidad recae sobre el consecuente en total dependencia del antecedente, de ahí la necesidad del planteamiento hipotético. De igual modo, a nivel gnoseológico, no podemos conocer la necesidad del consecuente, si no conocemos antes el antecedente. Por lo que, se trata de una necesidad únicamente cognoscible $a$ posteriori. Es evidente que es en la necesidad hipotética de las causas donde podemos encontrar un paralelo con la necesidad del origen kripkeana.

En el capítulo noveno del libro B de la Física, Aristóteles se plantea si la necesidad de la materia en los procesos naturales se da simplititer ( $\dot{\alpha} \pi \lambda \tilde{\omega} \varsigma$ ) o en términos hipotéticos $(\dot{\varepsilon} \xi \dot{v} \pi \circ \theta \varepsilon \dot{\varepsilon} \sigma \varepsilon \omega \varsigma)^{54}$. Esta discusión va dirigida contra dos modos de entender el desarrollo de los procesos naturales. El primero de ellos es representado por los $\varphi v \sigma i o ́ \lambda o \gamma o 1$, especialmente Anaxágoras y Empédocles, que mantenían la tesis según la cual los procesos y las disposiciones de los entes naturales se daban mecánicamente, por la sola eficacia causal de la materia ${ }^{55}$. El segundo de los modos

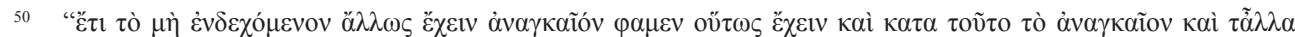

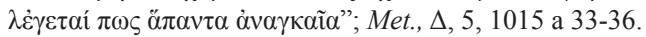

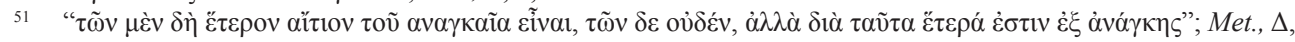
5,1015 b 9-11.

52 Thom. In metaph., V, 833; cfr. Thom. In phys. II, 15, 270.

53 Thom. In metaph. V, 834; cfr. Thom. In Phys. II, 15, 270. Vid. Sorabji, R. Necessity, Cause and Blame. Perspectives on Aristotle's Theory, Cornell University Press, Ithaca-New York, 1980, pp. 143-154.

54 Phys., B, 9, 199 b 34-35. Para un buen comentario del sentido general de Physica B 9, vid. Quarantotto, D. Causa finale, sostanza, essenza in Aristotele. Saggio sulla struttura dei processi teleologici naturali e sulla finzione del telos, Bibliopolis, Napoli, 2005, pp. 178-212.

55 Phys., B, 8, 198 b 10-32. 
viene representado por aquellos que mantienen que los procesos naturales se dan por puro acaso. Esta última posición, si bien no es mencionada en el capítulo que nos ocupa, ha sido objeto de tratamiento en los capítulos precedentes ${ }^{56}$. Teniendo en cuentas estas dos posiciones antitéticas, Aristóteles explora dialécticamente la posibilidad de una tercera vía que suponga un cierto justo medio entre el determinismo mecanicista y el puro indeterminismo del azar. Los procesos naturales ¿son absolutamente necesarios, o absolutamente contingentes? Pues bien, habiendo ya despachado la posibilidad de que los procesos sean azarosos, Aristóteles se ocupa ahora de la tesis determinista.

La necesidad de la materia para los procesos naturales es siempre de naturaleza hipotética y no absoluta ${ }^{57}$. Pues, si fuera debida a un principio intrínseco a la materia, la imagen de los procesos sería la de un mecanicismo que se desenvuelve por las propiedades inherentes a la materia, lo que Aristóteles se asegura de mostrar como algo absurdo:

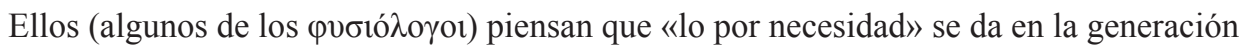
igual que si alguien creyera que un muro se origina necesariamente del hecho de que lo pesado se dirige por naturaleza hacía abajo y lo ligero hacia la superficie: de modo que las piedras y los cimientos van abajo, la tierra arriba por su ligereza y los maderos arriba del todo pues son los más ligeros ${ }^{58}$.

Decir lo anterior es, según el Estagirita, como afirmar que la generación y la disposición final de las partes de una casa es necesariamente así por las propiedades específicas de los materiales con los que está hecha. De tal forma que los materiales con mayor masa queden abajo y los de menor arriba, tal y como ocurre con el aceite y el agua que, lejos de mezclarse, se disponen y organizan en función de sus densidades. Más bien, por tanto, la necesidad de la materia habrá de entenderse de modo hipotético. Pero creo que aquí Aristóteles divide la cuestión en dos momentos diversos, aunque relacionados. En primer lugar, la materia, en general y en particular, es necesaria para que exista una substancia determinada. Para toda substancia el substrato o materia es condición necesaria pero no suficiente de su existencia:

Y sin embargo, por más que el muro no se origina sin éstos, tampoco lo hace por causa de éstos -si no es como materia- sino para ocultar y salvaguardar ciertas cosas. Igualmente

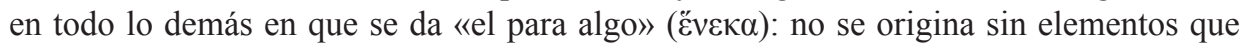
tienen como necesaria su naturaleza, pero, desde luego, no se origina por causa de éstos -si no es como materia- sino «para algo»: por ejemplo, ¿por qué la sierra es precisamente así? A fin de que sea «esto precisamente» y «para esto precisamente» ${ }^{59}$.

Esto es, este muro no se origina sin ladrillos, pero eso no quiere decir que el muro se origine solo ni principalmente a causa de éstos, sino que, en el proceso de generación, la causa que rige es la causa final, pues es con vistas a ella que se disponen los elementos materiales sin los cuales el existente no podría darse. De

56 Phys., B, 4-6.

57 Cfr. Quarantotto, D. "Ontologia della causa finale Aristotelica”, Elenchos, Rivista di studi sul pensiero antico, $\mathrm{n}^{\mathrm{o}}$ 22, fasc. 2, 2001, pp. 329-365.

58 Phys., B, 9, 199 b 35-200 a 5.

59 Phys., B, 9, 200 a 5-11. 
igual modo, la sierra no se origina sin el hierro, pero dado el hierro no se origina, sin más, la sierra. Sino que, más bien, la materia deviene una sierra a fin de que sea precisamente una sierra, un artefacto para dividir. Por tanto, de momento, tenemos:

a) Dado un artefacto (en general) necesariamente su materia (en general).

b) Dado esta mismísima sierra necesariamente su mismísima materia.

Pero la inversa no es verdadera:

a') Dada una materia necesariamente un artefacto.

b') Dada esta mismísima materia necesariamente esa mismísima sierra ${ }^{60}$.

Hasta aquí, Aristóteles está proclamando sencillamente que, para todo existente generado, en este caso cualesquiera objetos productos del arte, su materia es ontológicamente necesaria, pues si existe el artefacto es porque ha sido generado a partir de algo que sigue existiendo él. En otras palabras, para todo generado, necesariamente el substrato, pues: "La generación es imposible si no preexiste algo. Así, pues, es evidente que por necesidad preexistirá alguna parte; la materia, en efecto, es tal parte (ya que está presente en la cosa y se hace de ésta)"61. Esta relación de necesidad hipotética puede entenderse en los términos de la potencia y el acto: dado un existente en acto, es necesaria la potencia desde la que emerja la actualización; dado el artefacto, necesariamente su materia.

Sin embargo, Aristóteles va más lejos y plantea otra necesidad de la materia. La materia no es sólo necesaria para que el individuo exista sino, y sobre todo, para que pueda cumplir su función: "Sin embargo, este «para lo cual» no podría darse si no fuera de hierro; luego es necesario que sea de hierro si va a ser una sierra y va a producirse su trabajo"62. En este caso, ya no es sólo que esta materia sea necesaria para la existencia de esta sierra, que ya existe como unidad materio-formal, sino que es necesaria también una determinada especie de materia, si bien en sentido amplio, si las propiedades de dicha especie de materia satisfacen suficientemente los pormenores del fin buscado. A fin de que esto sea precisamente una sierra, que es para cortar, es necesaria una materia que soporte el fin, que tenga la potencia para soportar el fin. Imaginemos una sierra de hielo, ¿es una sierra, en sentido pleno? Creo que podríamos llamarla sierra, pero sólo por su aspecto. Parece la herramienta a la que llamamos sierra y, por eso, usaríamos el mismo nombre, pero nadie la consideraría una herramienta que sirve para cortar. Más bien diríamos que es una escultura de hielo con aspecto de sierra. Y, al que la hizo, no lo llamaríamos herrero, sino escultor de arte efímero. Por tanto, una vez más, la necesidad de la materia está siempre condicionado por el fin y el qué es de una cosa. La especie de la materia, elegida por sus potencialidades específicas, es necesaria en función del fin y éste es de lo que depende si aquello es una sierra o un objeto artístico. Por tanto, por último tenemos:

c) Dada una sierra necesariamente la especie de la materia que tenga la potencia de soportar el fin.

Y no:

c') Dada una especie de materia que sea potente para determinados fines necesariamente una sierra.

60 Si quisiéramos ahondar más en la cuestión, lo que si sería verdadero es decir que: «dada esta mismísima materia esa mismísima sierra es necesariamente posible». O sea, si B es la materia y A la sierra, la inversión de $\mathrm{A} \rightarrow \square$ B sería B $\rightarrow \square \diamond \mathrm{A}$, y no B $\rightarrow \square$ A. Vid. Met. $\Theta, 4,1047$ b 14-30; cfr. García Marqués, A. "Potencia, finalidad y posibilidad en «Metafísica» IX, 3-4”, Anuario Filosófico, vol. 23, n 2, 1990, pp. 147-159.

61 Met., Z, 7, 1032 b 30-1033 a 1.

62 Phys., B, 9, 200 a 11-13. 
Aristóteles continúa el pasaje justificando a qué se debe la necesidad hipotética de la materia. Para ello, introduce una interesante analogía con la geometría. En efecto, allí la necesidad es también hipotética, pero el esquema es exactamente el inverso. $\mathrm{Si}$, análogamente, la línea recta es la materia a partir de la cuál se constituyen los triángulos, resulta que: "Dado que la línea recta es precisamente así, necesariamente el triángulo tiene sus ángulos iguales a dos rectos; pero no porque esto último sea así, será así también la línea recta"63. En otras palabras, en matemáticas, los elementos que constituyen la materia de los objetos geométricos son aquellos que "generan" las figuras con sus propiedades necesarias. Dada la materia los objetos serán necesariamente así, pero no al revés. A este respecto, Tomás de Aquino lleva a cabo una importante observación:

Dice primero que de algún modo lo necesario en las ciencias demostrativas acontece de modo semejante al de las cosas que se generan naturalmente. Pues en las ciencias demostrativas lo necesario se da por lo anterior; es decir, puesto que la definición de ángulo recto es tal, es necesario que el triángulo sea tal que tenga tres ángulos iguales a dos rectos. De aquello primero que es tomado como principio, proviene necesariamente la conclusión ${ }^{64}$.

Esto es, si $F$ es el fin, y $M$ la materia, entonces, en geometría, « $M$ (entitativamente anterior) $\rightarrow \square F$ (entitativamente posterior)», siendo la materia la que aporta la razón de necesidad. Sin embargo, en la generación el esquema queda invertido: $F$ (entitativamente posterior) $\rightarrow \square M$ (entitativamente anterior), siendo el fin la ratio de la necesidad de la materia ${ }^{65}$. Así, comenta El Aquinate: "En las cosas que se hacen por algo, según el arte o la naturaleza, se da la conversión; porque si el fin fuera, o es, es necesario que vaya a ser o sea aquello que es antes del fin. Si aquello que es anterior al fin no es, tampoco será el fin" ${ }^{\text {" }}$. Con esta aclaración se entiende perfectamente lo que Aristóteles escribe a continuación. Colocamos entre paréntesis algunas aclaraciones:

En cambio, en las cosas que se originan «para algo» sucede inversamente: si va a haber (porque la generación está en proceso) o hay un fin (el objeto pleno), también (necesariamente) habrá o hay lo que antecede (su materia) (...). De modo que si va a haber una casa (o ya la hay), necesariamente tiene que haber, o tiene que producirse, estas cosas; o, en general, tiene que haber la materia que es «para algo» (potente con respecto a un fin) -como los ladrillos y piedras- si va a haber una casa. Sin embargo, el fin no es «por causa de» éstos -si no es en tanto que materia- ni será por causa de éstos. En general, si no existen éstos no habrá casa ni sierra; la una, si no hay piedras, la otra si no hay hierro ${ }^{67}$.

Esto es, que la materia que está en el origen de un artefacto (esta mesa, este atril, este muro, esta sierra) es condición necesaria pero no suficiente para la existencia de dicho artefacto. Por ello, existiendo el artefacto, la mismísima materia a partir de la cual fue hecha le pertenece necesariamente, si bien no como propiedad, sino

\footnotetext{
Phys., B, 9, 200 a 16-18.

Thom. In Phys. II, 15, 273.

Thom. In Phys. II, 15, 270 in fine.

Thom. In Phys. II, 15, 273.

Phys., B, 9, 200 a 19-29.
} 
como causa, y siempre en función del fin y la forma del objeto generado ${ }^{68}$. Por ello, concluirá Aristóteles, el filósofo natural debe tratar ambas causas, la material y la formal/final, pero más esta última, porque el fin es causa de la materia, pero no a la inversa: pues el fin no es tal porque la materia sea tal, sino más bien que la materia es tal porque el fin es tal.

\section{Conclusiones}

Ciertamente son muchas las cosas que se han visto en este artículo. Por ello, merece la pena una recapitulación a modo de conclusión. En primer lugar, hemos propuesto una hipótesis interpretativa acerca del origen de la denominación «esencialismo aristotélico». Es muy probable que, o bien sea una etiqueta generada de espaldas a Aristóteles, o bien se deba a una desafortunada interpretación de la predicación per se y la predicación per accidens en términos de propiedades esenciales y propiedades accidentales. Como hemos mostrado, Aristóteles, bajo ningún concepto, considera que la esencia ( $\tau$ ò $\tau$ í $\tilde{\eta} v$ cĩval) de un individuo sea equivalente al conjunto de las propiedades necesarias del mismo. Entre otras cosas, aceptar esto es considerar que la esencia es algo que el individuo tiene, no aquello que el individuo es.

En segundo lugar, nos hemos esforzado en encontrar una vía que en la que la noción de esencia kripkeana puede ser asumida por una genuina posición aristotélica. Sin duda, creemos haber hallado una estructura teórica apropiada en la doctrina de las cuatro causas, en su planteamiento ex hipothesi. En otras palabras, lo que para Kripke son propiedades necesarias de re, son para Aristóteles las causas necesarias con respecto a una substancia ya en acto. En efecto, esta es la marca distintiva de la necesidad hipotética tal y como el Estagirita la plantea en diversos lugares de su obra.

En tercer lugar, y en especial, la denominada necesidad del origen material de los artefactos defendida por Kripke, resulta estructuralmente análoga a la necesidad hipotética de la causa material. A continuación, vamos a presentar un bosquejo unitario de este asunto:

i. La necesidad hipotética se da entre causas extrínsecas y diversas, de tal modo que una de ellas adquiere razón de necesidad en total dependencia de la existencia efectiva de la otra. Así, la materia es necesaria con respecto a un fin/forma en acto.

ii. Esta relación existente entre ambas causas ha de estar fundada en una relación real, que indique que una es, al menos en parte, realmente responsable de su efecto. Esto es, la materia ha de cumplir un papel causal efectivo en la realización del artefacto pleno, o sea, que sea de facto el substrato a partir del que algo se hace y del que algo está hecho.

iii. Las causas, en su planteamiento hipotético, siempre guardaran una relación tal que el antecedente será siempre lo entitativamente posterior y el consecuente, que sustenta la razón de necesidad, será siempre lo entitativamente anterior.

iv. De todo lo anterior se desprende la necesaria cognoscibilidad a posteriori de esta relación real de necesidad. Pues, sólo conociendo el antecedente conocemos la necesidad del consecuente.

v. Por otro lado, nótese que la necesidad en el planteamiento hipotético, nace de la

68 Phys., B, 9, 199 b 34-200 a 14. 
consideración de aquellas entidades contingentes, no separadas, que pueden ser y no ser. Por ello, tanto Aristóteles como Kripke, se aferran a ejemplos que incluyen entes por naturaleza y entes por arte. De este modo, al plantear esta necesidad operamos ex supositio: suponemos que algo existe, pues, si existe, necesariamente existen o han existido sus causas como condiciones necesarias.

vi. Por último, el planteamiento hipotético resulta siempre la expresión de condiciones necesarias pero no suficientes para la existencia de un individuo. Pues, existiendo los ladrillos no necesariamente habrá una casa.

De (i-iii) queda reflejada la naturaleza metafísica de la razón de necesidad; (iv) supone el estatuto gnoseológico de este tipo de necesidad; (v) y (vi) resultan dos consideraciones generales acerca del carácter hipotético de esta relación entre causas. Si todo esto es correcto, creemos haber mostrado cómo las intuiciones que sostienen, en especial, la necesidad del origen material de los artefactos defendida por Kripke son perfectamente justificables desde el aristotelismo.

\section{Referencias bibliográficas}

\section{Fuentes primarias y traducciones}

Aristotele. Organon, ed. Maurizio Migliori, testo greco a fronte, Bompiani, Milano, 2016.

Aristóteles. Física, trad. José Luis Calvo Martínez, texto a fronte, CSIC, Madrid, 1996.

Aristóteles. Metafísica, ed. trilingüe de Agustín García Yebra, Gredos, Madrid, 1998.

Aristóteles. Tratados de lógica (Organon), vol. I y II, trad. de Miguel Candel Sanmartín, Gredos, Madrid, 2014.

Aristotle. Physics: Books I and II, trans. by William Charlton, Oxford University Press, Oxford, 1979.

Aristotle. Posterior Analytics, Topica, ed. LOEB, Harvard University Press, Massachusetts, 1960.

Aristotle. Posterior Analytics, trans. by Jonathan Barnes, Clarendon Press, Oxford, 1994.

Aristotle. Topics. Books I and VIII with excerpts from related texts, by Robin Smith, Clarendon Press, Oxford, 1997.

Kripke, S. Naming and Necessity, Harvard, Cambridge, 1980; originalmente publicado como Kripke, S. "Naming and Necessity" en Davidson D., Harman G. (eds.). Semantics of Natural Languages, Reidel, Dordrecht, 1872, pp. 253-355. Citamos por la reedición de la versión de 1980: Kripke, S. Naming and necessity, Blackwell, Oxford, 2013 y usamos la traducción de Kripke, S., El nombrar y la necesidad, tr. Margarita M. Valdes, Universidad Autónoma de Mexico, Mexico, 2005.

Kripke, S. "Identity and Necessity", en Munitz, K., Milton K. (eds.). Identity and Individuation, New York University Press, New York, 1971, pp. 135-164. Citamos por su reedición: Kripke, S. Collected papers, vol. 1, Philosophical troubles, Oxford University Press, Oxford, 2013, pp. 1-26. Usamos como traducción: Kripke, S. "Identidad y necesidad", tr. Margarita M. Valdes, Cuadernos de Crítica, $\mathrm{n}^{\mathrm{o}}$ 7, Universidad Autónoma de México, $\mathrm{n}^{\circ}$ 7, México, 1978, 47 pp.

\section{Bibliografía secundaria}

Aquinatis, Th. In metaphysicam aristotelis commentaria, Marietti, Taurini, 1926.

Aquinatis, Th. In octo libros physicorum aristotelis expositio, Marietti, Taurini, 1954. Edición castellana: Aquino, Tomás, Comentario a la Física de Aristóteles, traducción, estudio 
preliminar y notas de Celina A. Lértola, EUNSA, Pamplona, 2011.

Berti, E. Aristotele. Dalla dialettica alla filosofia prima con saggi integrative, Bompiani, Milano, 2014.

Berti, E., Rossitto, C. Aristotele. Il libro primo della «Metafisica», Laterza\&Figli Spa, RomaBari, 1993.

Cohen, S. M. "Essentialism in Aristotle", Review of Metaphysics, n 31, 1978.

Forbes, G. The Methaphysics of Modalitiy, Clarendom Press, Oxford, 1985.

Frede, M, Patzig, G. Il libro Z della Metafisica di Aristotele, Vita e pensiero, Milano, 2001.

García Marqués, A. "Tò tí ên eînai, tò tí esti, tò ón: su sentido y traducción”, Convivium, 2017 (en prensa).

García Marqués, A. "Potencia, finalidad y posibilidad en «Metafísica», IX, 3-4", Anuario Filosófico, vol. 23, n 2, 1990, pp. 147-159.

García-Lorente, J. A. "La ciencia de los principios y de las causas primeras", Anales del Seminario de Historia de la Filosofía, vol. 33, n 1, 2016, pp. 11-31.

Kirwan, Ch. "How strong are the objections to essence?". Proceedings of the Aristotelian Society, vol. 71, 1970-1971.

Kung, J. “Aristotle on Essence and Explanation”, Philosophical Studies, no 31, 1977.

Natali, C. "Aitia in Plato and Aristotle, from everyday language to technical vocabulary", en Natali, C., Viano, C., Zingano, M. (ed.). Aitia I, Les quatre causes d'Aristote. Origines et interpretation, Editions Peeters SA, Leuven, Belgium, 2013, pp. 39-73.

Pérez Otero, M. Esbozo de la filosofía de Kripke, Montesinos, Barcelona, 2015.

Quarantotto, D. "Ontologia della causa finale Aristotelica”, Elenchos, Rivista di studi sul pensiero antico, $\mathrm{n}^{\circ} 22$, fasc. 2, 2001, pp. 329-365.

Quarantotto, D. Causa finale, sostanza, essenza in Aristotele. Saggio sulla struttura dei processi teleologici naturali e sulla finzione del telos, Bibliopolis, Napoli, 2005.

Reale, G. Introduzione, traduzione e comentario della «Metafísica» di Aristotele, testo greco e a fronte, Bompiani, Milano, 2009.

Sorabji, R. Necessity, Cause and Blame. Perspectives on Aristotle's Theory, Cornell University Press, Ithaca-New York, 1980.

White, N. “The origins of Aristotle's essentialism”, Review of Metaphysics, n 26, 1972.

Witt, Ch. "Aristotelian Essentialism Revisited", Journal of the History of Philosphy, n' 27-2, 1989.

Witt, Ch. Substance and essence in Aristotle: an interpretation of "Metaphysics VII-IX", Cornell University Press, New York, 1989. 